\title{
The Design and Implementation of Workflow Simulation based on the Theory of Discrete Event
}

\author{
Kehe $\mathrm{Wu}^{1, \mathrm{a}}$, Shuo Shi ${ }^{1, \mathrm{~b}}$, Gang Ma ${ }^{1, \mathrm{c}}$ and Shengzhuang Wang ${ }^{1, \mathrm{~d}}$ \\ ${ }^{1}$ School of North China Electric Power University, Beijing, China \\ a epuwkh@126.com, ${ }^{b}$ qq150238517@126.com, ' hdmagang@163.com, 'ncepuwangsz@yahoo.cn
}

Keywords: Workflow, Simulation, Discrete Event.

\begin{abstract}
With the development of workflow technology, operation simulation of workflow is becoming a new hot spot of the workflow technology. In order to further promote the construction work of the enterprise standardization and process. The present study establishes a workflow operation simulation model based on discrete event dynamic system theory and designs simulation methods for this model based on events scheduling. The simulation clock applies the event-oriented promoting way and inverse transform method in the generation of random variables. Finally, we give an example of process simulation.
\end{abstract}

\section{Introduction}

From the view of workflow system applications, the dynamic analysis of the model has a practical significance on business process. But it is still a fragile link in the workflow system simulation. The difficulty lays on building up the simulation model and analyzing simulation data which requires professional knowledge of statistics. However, the workflow system is unsafe when it is in lack of the support for simulation theory and simulation tools. Therefore, the workflow simulation technology has become the hot spot of the current workflow technology.

There are three methods including ICAM(Integrated Computer Aided Manufacturing) Defination3(IDEF3) method, Petri net method [1,2,3,4]and workflow method [5,6,7,8]on workflow model simulation. But the first two methods make it impossible to analyze and implement model. Therefore, we establish a simulation model for the workflow and give details of the key features of the method to achieve the desired results.

The paper first introduces the recent research status of the workflow simulation model, and then introduces the principle based on discrete event system simulation. Finally, it gives the simulation model and its implements of the workflow management system.

\section{The simulation theory of the discrete event system}

The discrete event system is a dynamic system [9] which is driven by event and whose system state changes occur in a series of discrete points of time. There are some important concepts in it: Entity [10], Event, Simulation clock, Activity, and Random number generator [9].

The simulation theory of the discrete event system. We regard events as the basic unit of the simulation system. Each Event should be related to an event handler program to deal with the effects that it takes on the entity and create a new follow-up event.

In the implementation of this method, all events are on the table of future events. Future events table is stored by all future events and their happening moment. Future event control components in the model select the events taken place at earliest moment from the table of future events and put the simulation clock to the time of the event, and then call the event handler program to execute it until the end of simulation [11,12].

The generation method of random variables. The simulation requires random variables with high accuracy and efficiency. There are inverse transform method, combination method, convolution method and acceptance-or-rejection method [13]. Here we use the most commonly used method-inverse transform method. 
Set the discrete random variable $\mathrm{x}$ related to the values $x_{1}, x_{2}, x_{3}, \ldots, x_{n}$ and their possibilities related to $p\left(x_{1}\right), p\left(x_{2}\right), p\left(x_{3}\right), \ldots, p\left(x_{n}\right)$, the condition is: $0<p\left(x_{i}\right)<1$, and $\sum_{i=1}^{n} p\left(x_{i}\right)=1$, the distribution function shown in Fig. 1.

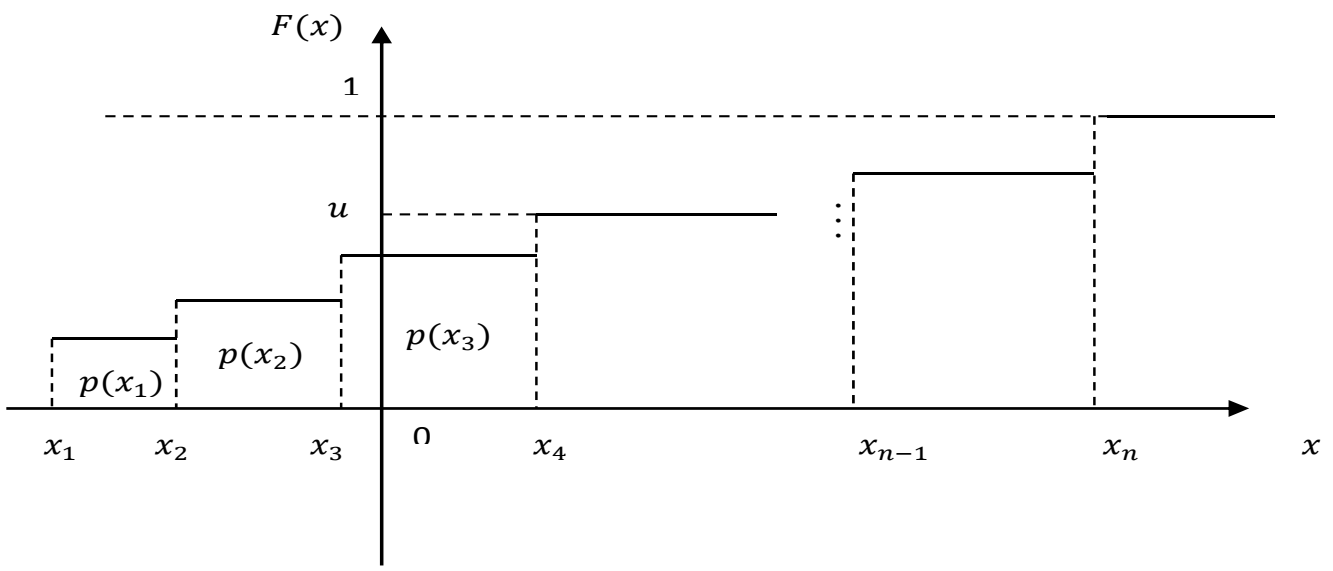

Fig. 1. Inverse transform method of discrete distribution.

In order to get the discrete random variables with inverse transform method, we put the intervals $[0,1]$ into $\mathrm{N}$ sub-intervals according to the values of $p\left(x_{1}\right), p\left(x_{2}\right), p\left(x_{3}\right), \ldots p\left(x_{n}\right)$.Then it can generate the distributed independent random numbers $\mathrm{u}$ in the interval[0,1]. According to which interval the value of $\mathrm{u}$ falls in, the corresponding random variable $x_{i}$ is what we need.

\section{The process model and method of workflow running simulation}

Combined with workflow engine characteristics of the workflow management system and simulation analysis of the discrete event systems, we set up the simulation process model shown in Fig. 2 for workflow running.

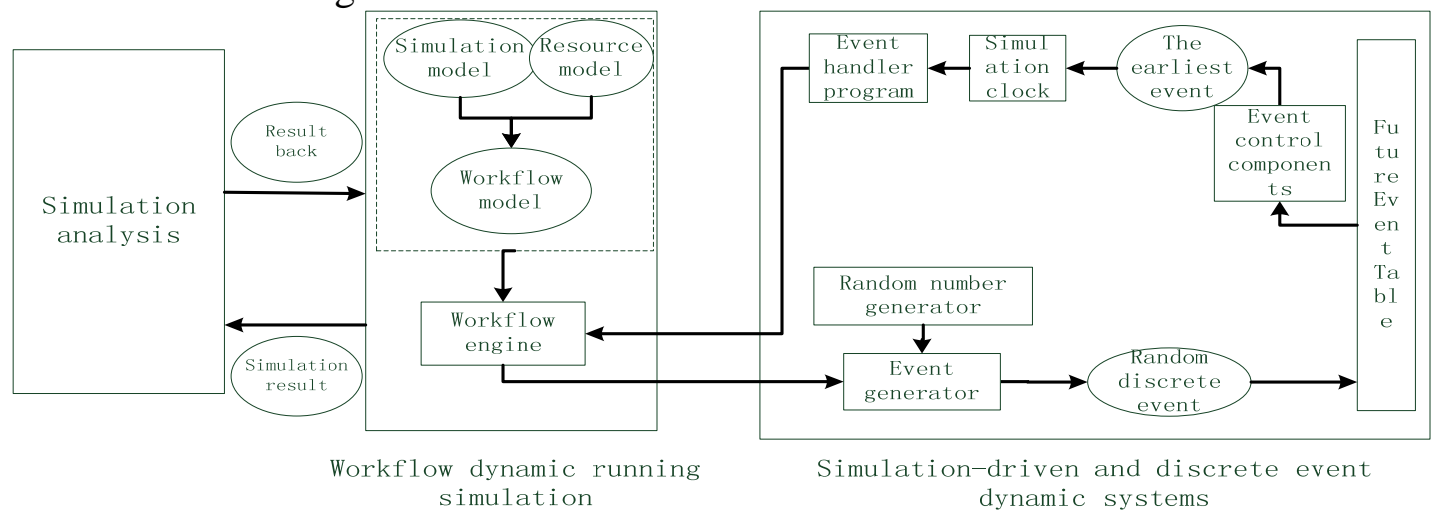

Fig. 2. the simulation process model for workflow running.

Simulation driver of discrete event system. Simulation driver of the discrete event system is the core of the process model for running simulation. The workflow engine finishes the automated operation of the workflow model with the help of simulation driver. The core work of simulation driver is to complete the schedule of future event table, including the following context.

Simulation clock. Advancing method of event-oriented: in a discrete event system, there is no change during intervals of two adjacent events. The method is to put the simulation clock directly from the happening time of an event to the next happening time over the "inactive" state. That is, each advancing is to select the earliest event from the future event table and then put the simulation clock to the happening moment. As the generation of events is random, so is the advancing speed.

Event control component and the future event table. Future event scheduling algorithm shown in Fig. 3. 


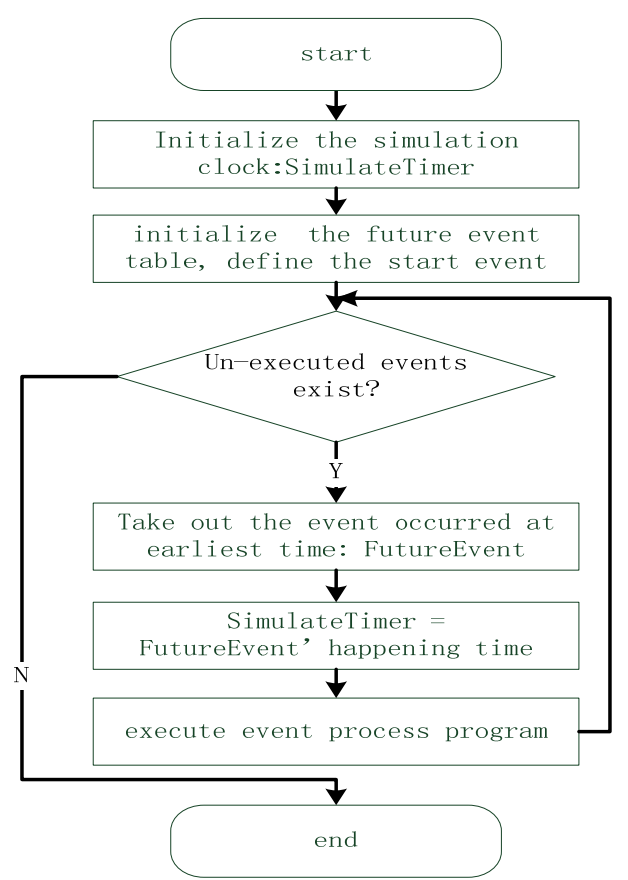

Fig. 3. Future event scheduling algorithm.

Random number generator and event generator. Random number generator plays a vital role in the running simulation stage. The workflow engine creates random discrete events with the help of random number generator.

Dynamic running simulation of workflow. Workflow dynamic simulation is one of the workflow execution transferring core units. Under the simulation actuation's function, it completes the workflow's automatic performance, and according to the node information, the flow transferring information, produces the new stochastic discrete event by the event generator.

The workflow model is the actual business process modeling by the process modeling tool, but the workflow process model is a static model which cannot be used for simulation directly. So we have to establish a workflow simulation model to get a more realistic simulation of the transferring process in reality. Workflow simulation model is an extension of the workflow model, adding the simulation information. Workflow management system supports multiple types of nodes, therefore, establishing the simulation model is corresponding to join the simulation information to these nodes and transitions, the following will discuss separately:

- Start node (Start), End node (End)

These two types of nodes are used to mark the beginning and the end of a business process, so there is no need to join simulation information to these types of nodes.

- Task nodes

This type of node represents the actual task, so we need to know the task execution time, resources and other information involved in the task. Among the processes in a workflow management system, mostly is the approval process in which less resource is used, so the article does not consider the process resource information. The time may be different each time you perform the actual task, so we get it by setting the time distribution and the corresponding parameters. The execution time here is composed of two parts: the waiting time after accepting the last task and the task processing time.

- Fork node, Join node

This type of Fork node is used to decompose a single branch into multiple parallel execution branches. The type of Join node is used to merge multiple branches into a single branch. As these two types of nodes only play the roles on controlling process running and when the process operation transfers to this type of node, automatic judgment executes, so we can ignore its implementation time, and there is no need to join other information to these types of nodes.

- Decision node

This type of node refers to node in which execution can be made automatically. When a process transfer to this type of node, expression results is determined automatically in accordance with the 
rules set previously and select the appropriate jump path based on the results. Therefore, we set the rules of the results of the distribution function and the corresponding parameters to obtain specific jump path. Each execution result corresponds to a jump path, therefore, the distribution of rules result can stand for the distribution of the jump path.

- Transition node

Transition represents the jump path between two nodes. Because the task node's Jump information is stored in the Transition and the path which is choose to jump is different every time and therefore we need to set an execution probability to each jump path. Under the function of the random number generator, the last automatically select the jump path.

\section{Implementation}

In this section, we give an example of process simulation. When workflow simulation runs, one simulation process will complete the following procedures in order: Select the flow chart, read into the information of flow, initialize the simulation clock, simulation execution, results analysis, we will illustrate this with the computer supplies requisitioned process which run procedures are shown in Table 1.

Table 1. Simulation process table.

\begin{tabular}{|c|c|c|c|c|c|c|}
\hline $\begin{array}{l}\text { Serial } \\
\text { number }\end{array}$ & Task content & transformer & $\begin{array}{l}\text { Processing } \\
\text { time }\end{array}$ & \multicolumn{2}{|l|}{ Time spent } & Completion \\
\hline \multirow{2}{*}{1} & \multirow{2}{*}{$\begin{array}{l}\text { The applicant fill out } \\
\text { the application form }\end{array}$} & \multirow{2}{*}{ A } & 2012-02-24 & \multirow{2}{*}{\multicolumn{2}{|c|}{$20 \quad 03$}} & \multirow{2}{*}{ processed } \\
\hline & & & $\begin{array}{lll}09 & 48 & 32\end{array}$ & & & \\
\hline \multirow{2}{*}{2} & \multirow{2}{*}{$\begin{array}{l}\text { Information operation } \\
\text { and maintenance } \\
\text { center signature }\end{array}$} & \multirow{2}{*}{ B } & 2012-02-24 & \multirow{2}{*}{\multicolumn{2}{|c|}{$\begin{array}{cc}08 & 03 \\
11\end{array}$}} & \multirow{2}{*}{ processed } \\
\hline & & & $\begin{array}{lll}17 & 51 & 43\end{array}$ & & & \\
\hline \multirow{2}{*}{3} & $\begin{array}{l}\text { Science and } \\
\text { technology }\end{array}$ & \multirow{2}{*}{$\mathrm{C}$} & 2012-02-25 & \multirow{2}{*}{\multicolumn{2}{|c|}{144}} & \multirow{2}{*}{ processed } \\
\hline & $\begin{array}{c}\text { information } \\
\text { department approval }\end{array}$ & & $\begin{array}{lll}02 & 05 & 47\end{array}$ & & & \\
\hline \multirow{2}{*}{4} & The person in charge & \multirow{2}{*}{$\mathrm{D}$} & 2012-02-25 & \multirow{2}{*}{46} & \multirow{2}{*}{4} & \multirow{2}{*}{ processed } \\
\hline & $\begin{array}{c}\text { of the library } \\
\text { confirmed }\end{array}$ & & $09 \quad 51 \quad 51$ & & & \\
\hline \multirow{2}{*}{5} & \multirow{2}{*}{ Forms archive } & \multirow{2}{*}{$\mathrm{E}$} & $2012-02-25$ & \multirow{2}{*}{\multicolumn{2}{|c|}{348}} & \multirow{2}{*}{ processed } \\
\hline & & & $\begin{array}{lll}18 & 25 & 59\end{array}$ & & & \\
\hline
\end{tabular}

\section{Summary}

Workflow simulation is the new topic of research in the field of workflow technology. This article establishes a workflow simulation model and designs the simulation method based on event scheduling for the model, and briefly describes the realization method of key functions. Finally we give the example of computer supplies requisitioned process to show workflow management system simulation processes and it reaches the expected effect.

The article needs to be improved as follows: First of all in the workflow management system, most of the processes are approval-type processes in which less resources used, so our article does not consider the process resource information, but only by adding time simulation; In addition, the process of interactive simulation capabilities also need to continue to study.

\section{References}


[1] Cheng Feifei, Wang Yaowu: Workflow structured modeling and simulation based on Petri Net for product design process. Journal of System Simulation, Vol.21 No.24(2009), p.7727 7731.

[2] Chen Xiang, Xia Guoping: Workflow modeling techniques based on Petri Net and its applications in simulation. Computer Engineering and Application, Vol.22(2003), p.84 86.

[3] Shen Mei, Liu TongYi et al.: Study on workflow modeling and simulating analyze based on high-level Petri Nets. Computer Engineering and Applications, Vol.32(2006), p.200 203.

[4] W.M.P. van der Aalst and A.H.M.ter Hofstede: Verfication of workflow task structure:A Petri-net-based approach Information Systems, Vol.25 No.1(2000), p43 69.

[5] Ji Haifeng, Fan Yushun: Simulating-oriented workflow model. Aeronautical Manufacturing Technology, Vol.1(2004), p49 51.

[6] Micrografx Inc. iGrafx Process User’s Guide. Microgragx Inc,1999

[7] Xia Yuan, Li Jianhua: Workflow simulation enviroment research based on historical data analysis. Computer Simulation, Vol.24 No.12(2007), p85 87.

[8] Chen Rui: Workflow dynamic simulation and model analysis based on XinPai Driven workflow model. Northwest University, 2009

[9] Gu Qitai: Modeling and simulation of discrete event system. Beijing: Tsinghua University Press, 1999

[10]Zhong Qi: Study and implementation of a hierarchal workflow simulation model.National University of Defense Technology, 2004, p19 23

[11] Workflow Management Coalition. Interface 1: Process Definition Interchange Process Model Wfmc-TC-1016-P,1999,http://www.wfmc.org

[12] Wang Weiping et.al.: Modeling and simulation of discrete event system. Beijing: Science Press, 2007

[13] Jiao Yanhua, Xu Hui et.al.: Method and simulation of producing a random variable. Electronic warfare, No.6(2004), p11 15 